\title{
Evaluation of Cytotoxic Effects of Different Concentrations of Porous Hollow Au Nanoparticles (PHAuNPs) on Cells
}

\author{
Smitha Rao, ${ }^{1}$ Chienwen Huang, ${ }^{2}$ Uday Tata, ${ }^{1}$ Peter Wu, ${ }^{3}$ Nikhil Arora, ${ }^{4}$ Jinsung Ahn, \\ Victor K. Lin, ${ }^{1}$ Yaowu Hao, ${ }^{2}$ and J.-C. Chiao ${ }^{1}$ \\ ${ }^{1}$ Department of Electrical Engineering, UT Arlington, 416 Yates Street, P.O. Box 19016, Arlington, TX 76019, USA \\ ${ }^{2}$ Department of Materials Science and Engineering, UT Arlington, 501 West 1st Street, P.O. Box 19031, Arlington, TX 76019, USA \\ ${ }^{3}$ Texas Academy of Math and Science, Denton, TX 76203, USA \\ ${ }^{4}$ Lamar High School, Arlington, TX 76012, USA \\ ${ }^{5}$ Arlington High School, Arlington, TX 76013, USA
}

Correspondence should be addressed to Smitha Rao; smitha@uta.edu

Received 31 July 2013; Accepted 27 October 2013; Published 19 January 2014

Academic Editor: Paresh Chandra Ray

Copyright (C) 2014 Smitha Rao et al. This is an open access article distributed under the Creative Commons Attribution License, which permits unrestricted use, distribution, and reproduction in any medium, provided the original work is properly cited.

\begin{abstract}
Nanoparticles (NPs) have been introduced as a suitable alternative in many in vivo bioapplications. The risks of utilizing nanoparticles continue to be an ongoing research. Furthermore, the various chemicals used in their synthesis influence the cytotoxic effects of nanoparticles. We have investigated the cytotoxicity of Porous Hollow Au Nanoparticles (PHAuNPs) on cancer cell lines PC-3, PC-3ML, and MDA-MB-231 and the normal cell line PNT1A. Cell proliferation for the different cells in the presence of different concentrations of the PHAuNPs was assessed after 24 hours and 72 hours of incubation using MTT assay. The study also included the cytotoxic evaluation of pegylated PHAuNPs. Identical cell seeding densities, particle concentrations, and incubation times were employed for these two types of Au nanoparticles. Our results indicated that (1) impact on cell proliferation was concentration dependent and was different for the different cell types without cellular necrosis and (b) cellular proliferation might be impacted more based on the cell line.
\end{abstract}

\section{Introduction}

Nanoparticles (NPs) exhibit unique properties compared to their constituent bulk materials. These include quantum confinement in semiconductor particles, surface plasmon resonance [1-8] in metal particles, and superparamagnetism in magnetic particles. Such unique properties allow the use of nanoparticles in a wide variety of applications. Nanoparticles such as fullerenes, liquid crystals, liposomes, Au nanoparticles, and quantum dots have been demonstrated in biomedical applications $[5,6]$, chemotherapy $[7,8]$, drug delivery [811], imaging [11] and cosmetics [12-14]. Gold nanoparticles (AuNPs) have been subjects of intensive research in the last decade. In vivo applications of AuNPs include photothermal ablation treatment and optical imaging. Imaging utilizes the surface plasmon resonance (SPR) effect of AuNPs, a strong enhancement of absorption, and scattering of light in resonant with the SPR frequency. AuNPs possess two additional important merits for in vivo applications: (1) they are generally considered as biocompatible; (2) they can be easily functionalized with well-established thiol-Au linkage. However, the SPR wavelength for solid AuNPs lies around $520 \mathrm{~nm}$, which excludes their in vivo applications since this wavelength is strongly absorbed by tissues and blood. In the last decade, several engineered Au nanoparticles such as nanoshells and nanocages have been developed to tune SPR wavelength of these nanoparticles to near-infrared region that is commonly regarded as a "clear window" for deep tissue penetration of light. Among these nanoparticles, Au nanoshells [15-23] consisting of $\mathrm{Au}$ shell and a dielectric silica core have been explored for bioimaging and therapy applications. Wang et al. [24] evaluated the use of gold nanoshells for real-time detection of biomolecular interactions in diluted blood. Low et al. [25] have studied nanoshellenhanced optical coherence tomography (OCT). Raman spectroscopy using Au nanoparticle-based contrast agents 




(a)

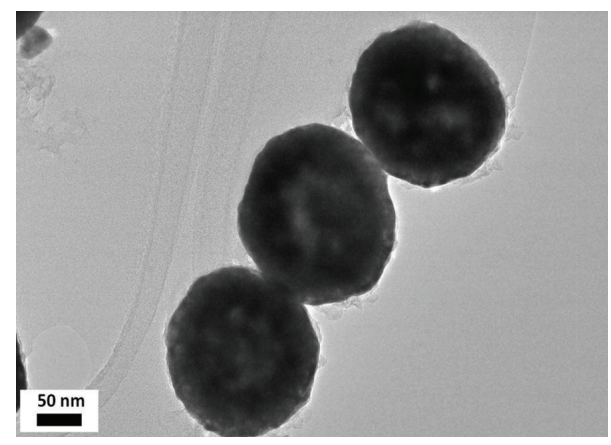

(b)

FIgure 1: The SEM (a) and TEM (b) images of the PHAuNPs were used to determine the sizes of the particles. It was found that the particle sizes were consistent at $150 \mathrm{~nm} \pm 10 \%$.

shows tremendous practical applications in biomedical imaging [26]. Jensen et al. [27] have demonstrated the use of gold nanoshells functionalized with a $\mathrm{pH}$-sensitive Surface Enhanced Raman Spectroscopy (SERS) reporter molecule responsive to the $\mathrm{pH}$ in the range of 3 to 7 . Keren et al. [28] showed SERS imaging using nanoshells. Another exciting application of $\mathrm{Au}$ nanoparticles is photothermal therapy; gold nanoparticles can be used to destruct bacteria and cancer cells by photothermal ablation [29-37]. It has been shown that localized heating and irreversible damage to tissue can occur in tumors treated with gold nanoshells [38-40]. Recently, Huang et al. [41, 42] demonstrated the PHAuNps having a sub- $20 \mathrm{~nm}$ porous shell with a $50 \mathrm{~nm}$ hollow core. A simple, surfactant-free process, termed as the "bubble template synthesis" method, has been developed and employed to synthesize those PHAuNPs. The unique morphology of the PHAuNPs allows strong absorbance and scattering of light in near-infrared wavelength due to the SPR effect. They have also demonstrated the attachment of Raman dyes to pegylated PHAuNPs to form "Raman nanotags," aiming for in vivo SERS cancer biomarker detection. The previous study included the cytotoxicity of the Raman nanotags using [3H] thymidine incorporation method. In this work, we present the cytotoxicity study (24 and 72 hours) of different concentrations of bare and pegylated PHAuNPs on normal prostate epithelium (PNT1A), prostate cancer (PC-3), lungmetastasized prostate (PC-3ML) cancer, and human breast carcinoma (MDA-MB-231) cell lines using the standard MTT assay.

\section{Materials and Methods}

2.1. Nanoparticles. Porous Hollow Au Nanoparticles (PHAuNPs) were synthesized using the bubble template syn thesis method detailed in the literature [43, 44]. Briefly, PHAuNPs were synthesized inside a three-electrode electrodeposition cell using $\mathrm{Ag} / \mathrm{AgCl}$ electrode in $\mathrm{NaCl}(3 \mathrm{M})$ solution acting as reference electrode with a platinum mesh counter electrode. Two anodic alumina filtration membranes having a pore diameter of $300 \mathrm{~nm}$ and thickness of $60 \mu \mathrm{m}$ were stacked and used to collect electrochemically generated hydrogen nanobubbles. The hydrogen nanobubbles were

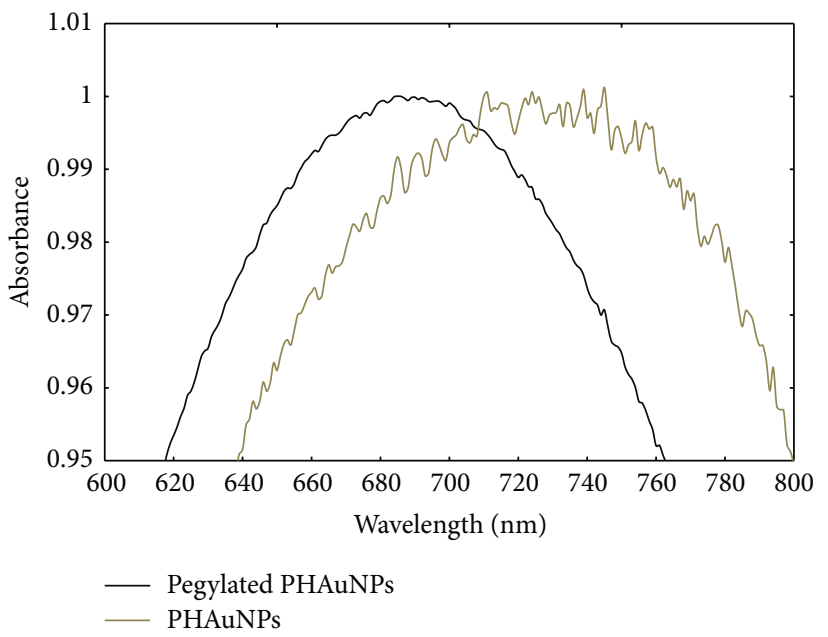

Figure 2: The extinction spectra of bare and pegylated PHAuNPs indicating a blue shift.

formed on the inner walls of the pores. The high concentration of hydrogen molecules at the bubble boundary reduced the $\mathrm{Au}+$ complex ion into metal $\mathrm{Au}$ to form Au clusters. These clusters in turn act as a catalyst that triggers an autocatalytical disproportionation reaction to form a gold shell around the hydrogen bubble. Metal Au evolved from clusters and particles to porous networks to form PHAuNPs when short reaction times of less than 10 minutes were allowed. In the case of the pegylated PHAuNPs, the nanoparticles were released by the dissolution of the alumina membrane using $2 \mathrm{M} \mathrm{NaOH}$ solution. The nanoparticles were cleaned by repeated dispersion in deionized water and centrifugation. The PHAuNPs were pegylated with $200 \mu \mathrm{M}$ SH-mPEG (MW $5 \mathrm{kDa}$ ) by an overnight stirring at $40^{\circ} \mathrm{C}$. The SEM and TEM images of the nanoparticles are shown in Figure 1.

The extinction spectra for the bare and pegylated PHAuNPs are shown in Figure 2. There is a blue shift for the pegylated particles. Also, the spectrum for the bare particles is broader. These may result from the possible aggregation of the bare particles. It is known that aggregation leads to 
a broader, red-shifted spectrum. It was also observed that while the colloidal suspension of the bare nanoparticles remained stable for up to a week with minimal aggregation, the pegylated PHAuNPs were stable for over four weeks without any visible aggregation.

The particle concentration of the suspension is determined by dissolving PHAuNPs into solution using Aqua Regia followed by inductively coupled plasma mass spectroscopy (ICP-Mass) measurement of the total amount of gold ions in the solution. The weight of the total Au obtained from the ICP-Mass and the particle size from SEM and TEM were used to calculate the number of particles in the suspension. Using the outer radii of the nanoparticles and an approximation of the inner radii, the molar concentration of one PHAuNP was calculated to be $8.22 \times 10^{-11} \mu \mathrm{mol}$. Thus, the number of nanoparticles in $1 \mu \mathrm{M}$ solution was nearly $12 \times 10^{6} / \mathrm{mL}$.

2.2. Tissue Culture. Prostate cancer cell line (PC-3) and human breast carcinoma cell line (MDA-MB-231) were obtained from American Type Culture Collection (ATCC, Manassas, VA). Lung-metastasized prostate cancer cells (PC3ML) were obtained from UTSW Medical Center (Dr. J. T. Hsieh, Department of Urology). Normal prostate epithelium cell line (PNT1A) was obtained from Sigma-Aldrich (SigmaAldrich, St. Louis, MO, USA). The cells were cultured in the culture media RPMI 1640 (Lonza, Walkersville, MD, USA) supplemented with $5 \%$ fetal bovine serum, $100 \mathrm{U} / \mathrm{mL}$ penicillin, and $100 \mu \mathrm{g} / \mathrm{mL}$ streptomycin under $5 \% \mathrm{CO}_{2}$ atmosphere at $37^{\circ} \mathrm{C}$.

\subsection{MTT Assay for PHAuNPs Effect on Cellular Proliferation.} The cells in exponential growing condition were trypsinized, hemocytometer-counted, and resuspended in growth media and seeded in 96 -well plates at $3 \times 10^{3}$ per $80 \mu \mathrm{L}$ per well. The cells were incubated under standard tissue culture conditions for 24 hours before PHAuNPs treatment. The bare and pegylated PHAuNPs fabricated as mentioned above were sterilized and prepared for use in the experiment. Briefly, the particles were centrifuged and pelleted at $13,200 \times \mathrm{g}$ for 5 minutes at room temperature, and re-suspended in $2 \mathrm{~mL}$ of $70 \%$ ethyl alcohol. This process was repeated twice. The particles were then re-suspended in growth media with vigorous vortex and sonication. The sterilized PHAuNP suspension was diluted to $4,20,100$, or $500 \mu \mathrm{M}$. Upon treatment, $20 \mu \mathrm{L}$ of the corresponding suspension was added to the designated cell containing wells to achieve final concentration of PHAuNPs at $0.8,4,20$, or $100 \mu \mathrm{M}$. Twenty microliters of media without PHAuNPs was also added to separate wells (with cells) as assay control (i.e., $0 \mu \mathrm{M}$ ). Wells with $100 \mu \mathrm{L}$ growth media, but without cells, were included as MTT assay background controls. Each treatment was in triplicates. The treated plates were then incubated for 24-hour period or 72hour period before being subjected to MTT assay procedures. The prepared PHAuNP suspensions were also incubated on Luria-Bertani (LB) agar plates confirming that the PHAuNPs used in the experiments were free of bacterial or fungal contamination (data not shown).
MTT based cell growth determination kit (SigmaAldrich, St. Louis, MO, USA) was used to assess the cellular proliferation potential after treatment of PHAuNPs. In brief, $10 \mu \mathrm{L}$ of MTT solution (10\% by volume of cell culture) was added to each well with 4 hours of incubation at $37^{\circ} \mathrm{C}$. One hundred microliters of the MTT solvent (equal to original culture volume) was added to the wells with additional incubation for 1 hour. The contents of each well of the 96well plate were transferred to eppendorf tubes and subjected to centrifugation at $13,200 \times \mathrm{g}$ for 10 minutes. One hundred microliters of the supernatant from each tube was carefully transferred to corresponding wells of a fresh 96-well plate and spectrophotometric (at wavelength of $570 \mathrm{~nm}$ with background at $690 \mathrm{~nm}$ ) readings were taken using Infinite 200 (Tecan Group AG, Switzerland). The results were expressed as the mean of the triplicated repeats after subtraction of the assay background. The proliferation potential of each treatment was compared after normalization to nontreated control to determine the effect of nanoparticles on cell growth.

\section{Results and Discussion}

In this study, we analyzed PHAuNPs' effects on cellular proliferation using PNT1A, PC-3, PC-3ML, and MB-MDA231 cell lines. After 24 hours or 72 hours of nanoparticle treatment, gross microscopic observation results indicated that there was no apparent cellular necrosis on all four cell lines compared to that of the non-treated controls. This suggests that the PHAuNPs may not have immediate cytotoxicity effects. Figure 3 shows a comparison between non-treated and treated PNT1A cells. In addition, it can be seen that the particles appear to have aggregated around the cells and on the plate substrate.

The MTT results of the impact of PHAuNPs at different concentrations on proliferation potential of PNT1A cells, an immortalized normal human prostate epithelium, are illustrated in Figure 4. At higher concentrations (i.e., $4 \mu \mathrm{M}, 20 \mu \mathrm{M}$, or $100 \mu \mathrm{M}), 24$ hours treatment of pegylated PHAuNPs appears to significantly enhance PNT1A cells' proliferation potential compared to the non-treated counterpart. However, $0.8 \mu \mathrm{M}$ of pegylated PHAuNPs does not show any significant effect. Interestingly, the proliferation enhancement peaks at nearly $80 \%$ over the control by treatment with $4 \mu \mathrm{M}$ pegylated PHAuNPs and gradually decreases with the increase in nanoparticle concentration. On the other hand, the bare PHAuNPs exhibit an inhibitory effect on PNT1A cells' proliferation at 24 hours in a concentration dependent manner with a significant difference observed at $100 \mu \mathrm{M}$ (more than $20 \%$ reduction of proliferation potential), suggesting that the coating of the PHAuNPs may play an important role in their interaction with the cells. Our results from extended treatment for 72 hours also indicated that both bare and pegylated PHAuNPs show a similar inhibitory effect on PNT1A cells (Figure 4). Furthermore, the inhibitory effect appears to be concentration dependent, as was seen in the case of the 24-hour bare PHAuNPs treatment.

The response of PC-3, prostate cancer cell line, to the PHAuNPs is depicted in Figure 5. MTT results indicated that 


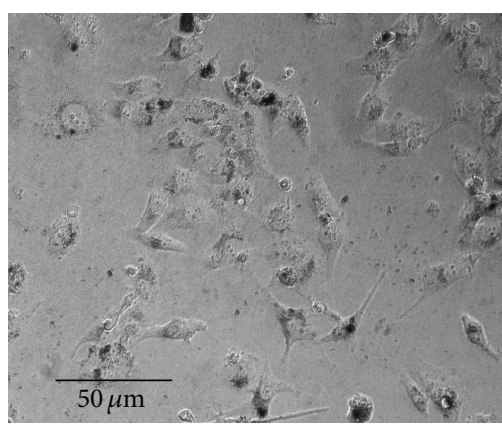

(a)

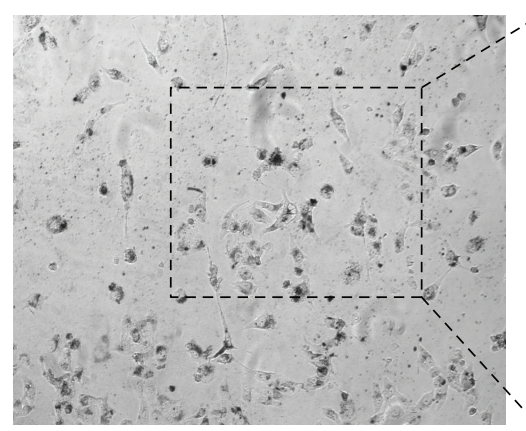

(b)

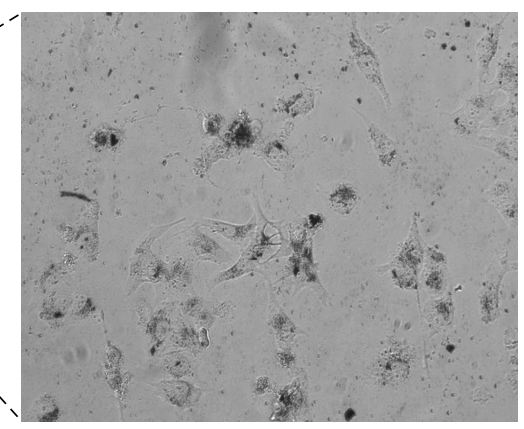

(c)

Figure 3: PNT1A cells (a) without PhAuNPs at 100x magnification and (b) with $4 \mu \mathrm{M}$ bare PHAuNPs at 100x magnification and (c) shows the distribution of the PHAuNPs in aggregation on the substrate and cells at 200x magnification.

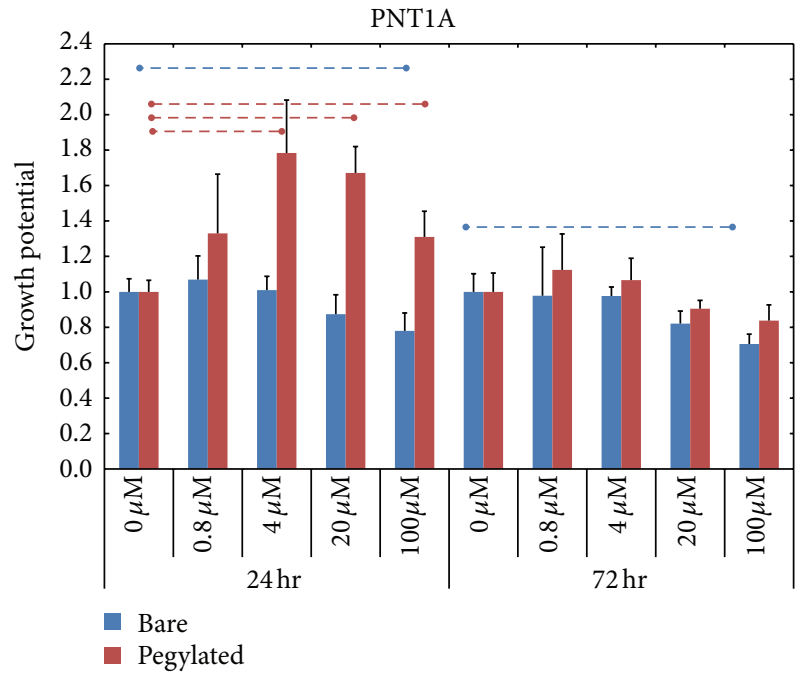

FigURE 4: PNT1A cell proliferation after treatment with bare and pegylated PHAuNPs for 24 and 72 hours. All the values depicted have been normalized to the non treated control $(0 \mu \mathrm{M})$. After 72 hours of treatment, there was a significant decrease in the proliferation of cells treated with $100 \mu \mathrm{M}$ bare nanoparticles. The horizontal bars indicate that there is a significant difference at $P \leq$ $5 \%$.

PHAuNPs treatment clearly produced an inhibitory effect on PC-3 proliferation. At $100 \mu \mathrm{M}$ of PHAuNPs, the inhibition is almost as high as $40 \%$ compared to the growth potential in the controls. This growth inhibition effect appears to be PHAuNPs concentration dependent. Interestingly, similar inhibitory patterns were observed from both bare and pegylated PHAuNPs treatment suggesting that the modification of PHAuNPs surface may not promote their interaction with PC-3 cells. Furthermore, no difference was observed either at 24 hours or at 72 hours suggesting that the response in PC-3 was immediate and sustainable.

Interestingly, PHAuNPs exhibit an enhancement in cell proliferation within 24 hours on PC-3ML cell line, a derivative of PC-3 cells with a higher metastatic potential, compared to the untreated counterparts as shown in Figure 6. This enhancement is inversely related to the PHAuNPs concentration, and it is more pronounced at 24 hours than at 72 hours.

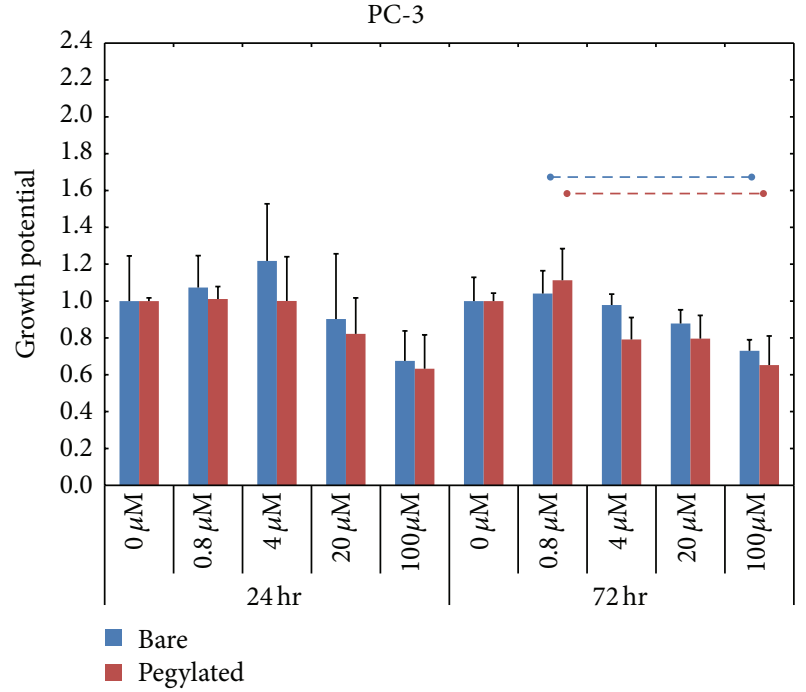

FIgURE 5: Proliferation of PC-3 after treatment with bare and pegylated PHAuNPs indicating inhibitory effect at $100 \mu \mathrm{M}$ after 72 hours in both cases.

In addition, unlike in PNT1A cells, the bare PHAuNPs appear to be more potent than the pegylated ones. These results suggest that the impact of PHAuNPs on cellular proliferation might not be directly linked to cell/tissue origin but is more cell line dependent.

Furthermore, an inhibitory effect of cell proliferation was observed in MDA-MB-231, an established breast cancer cell line, as shown in Figure 7. The inhibition patterns are similar to that observed in PC-3 cells (Figure 5).

Recent studies have implicated that nanoparticles may be applicable in cancer intervention $[5,6,8,9]$. Due to the difference in the physical properties of the nanoparticles in relation to their composite bulk material, the interaction of the particles on basic cellular functions has not been fully explored. Our study here provides information on the impact of nanoparticles on cell proliferation to aid in the understanding of the mechanism of interaction of nanoparticles with cells. We have chosen different cells as well as different 


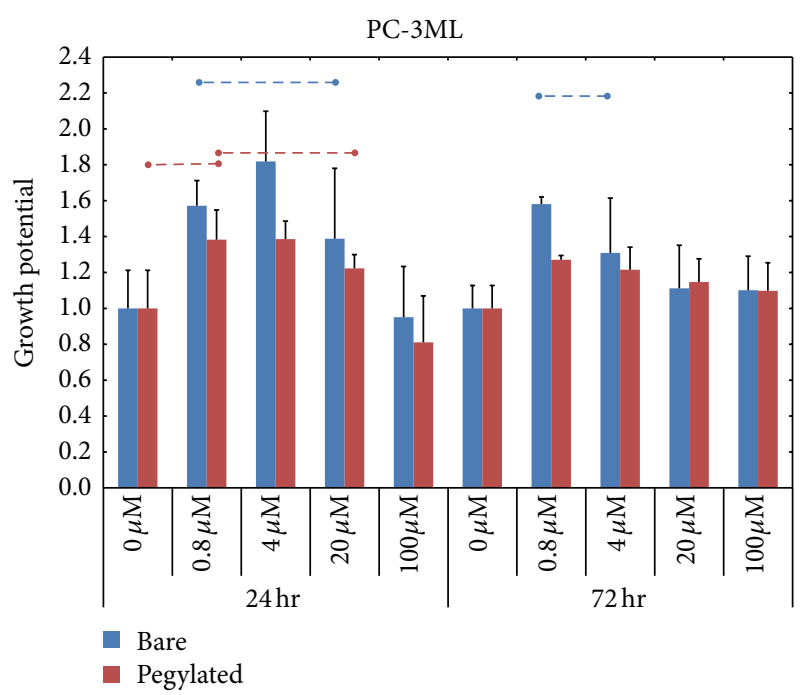

FIGURE 6: Proliferation of PC-3ML after treatment with bare and pegylated PHAuNPs. There was no significant change in cell proliferation after 72 hours of treatment for both types of PHAuNPs.

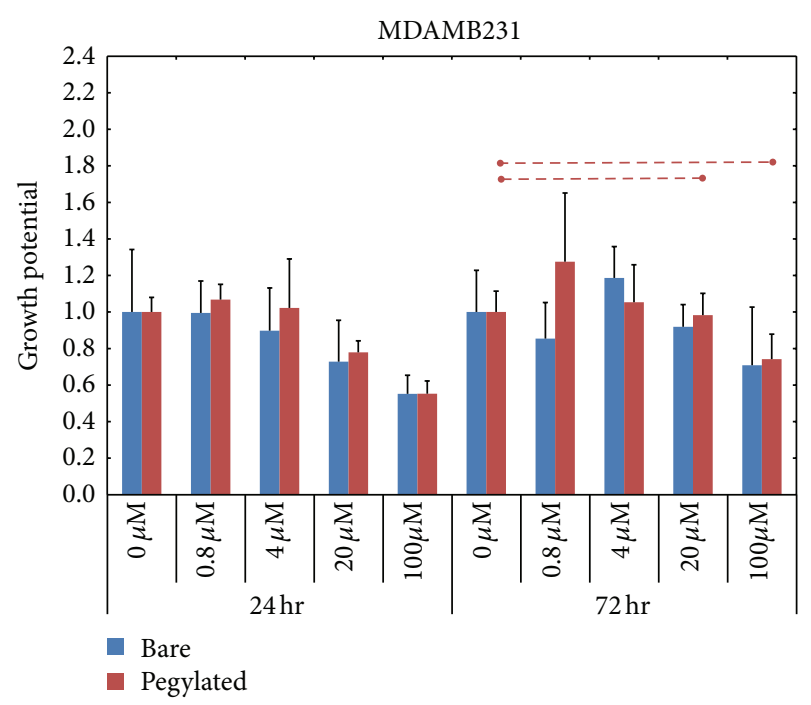

FIGURE 7: Proliferation of MDA-MB-231 after treatment with bare and pegylated PHAuNPs indicated that the effect was significant only in the case of the pegylated PHAuNPs at $20 \mu \mathrm{M}$ and $100 \mu \mathrm{M}$ after 72 hours of treatment.

concentrations of bare and pegylated PHAuNPs to study their effect on cells.

Previous studies have reported that the size of the nanoparticles may impact cell proliferation $[45,46]$. The size of the nanoparticles used in the current study was uniform at $150 \mathrm{~nm} \pm 10 \%$ (Figure 1). Therefore, we believe that the difference in cell proliferation observed was primarily due to the concentration of the nanoparticles. In addition, our results indicated that higher concentration of the nanoparticles tended to have more impact leading to further decrease in cell numbers compared to lower concentrations thereby supporting this notion. Thus, there appears to be a concentration dependent effect of nanoparticles on cell proliferation. These trends are observable after 24 hours as well as 72 hours.

The more pronounced effect in PC-3 compared to PNT1A may imply that the nanoparticles impact the cancer cell lines more severely than the normal cells. The observations also indicated that the impact of the nanoparticles is cell line dependent. This can be seen from the fact that PC-3 cells was more severely affected than normal cells and the effect on MDAMB231 cells was different compared to the other cells tested. There is also an indication that the effect of the nanoparticles was more evident after 72 hours, suggesting that more than 24 hours were required for the particles to take effect. Interestingly, in the case of MDA-MB-231, the impact was overly enhanced within 24 hours when compared to PC-3, with continued incubation providing no further effect. The absence of a significant effect on PC-3ML after 72 hours further supports our earlier observation that the outcomes are cell line dependent.

Our data indicated that there was no clear difference between bare PHAuNPs and the pegylated PHAuNPs or Raman nanotags. Our results also indicated that more tumorigenic PC-3ML experienced a growth enhancement from the nanoparticles compared to its less potent parental line, PC-3. This further suggests that the cancer characteristics of a cell may not be the only factor related to the impact of the nanoparticles on cell proliferation. Also, our study was conducted over 24-hour period and 72-hour period in triplicates, using same initial seeding density to address the issue of the effects of seeding density in testing cytotoxicity of nanoparticles [46]. It has also been reported that the presence of sodium citrate on the surface of gold nanoparticles increases cytotoxicity [47]. The PHAuNP's tested did not contain any sodium citrate. Thus, our experiments and observations indicated that both the bare and pegylated PHAuNPs seem to have some inhibitory effect but prove to be noncytotoxic. Overall, it appears that the addition of nanoparticles tends to only decrease cell proliferation in certain cell lines such as PC-3, MDA-MB231, and PNT1A, while enhancing cell proliferation in PC-3ML.

\section{Conclusions}

In this paper we have studied the impact of bare and pegylated Porous Hollow Gold Nanoparticles on the proliferation of different cell lines. PNT1A, PC-3, PC-3ML, and MDAMB231 cells were treated with $0,0.8,4,20$, and $100 \mu \mathrm{M}$ of the bare and pegylated PHAuNPs in triplicates over periods of 24 and 72 hours. The results indicated that both types of particles at all concentrations were not cytotoxic. However, there was some growth inhibition at higher concentrations in the case of PC-3, PNT1A, and MDAMB231 whereas there was growth enhancement in PC3-ML after 72 hours. Thus, the impact of the nanoparticles appears to be time, concentration, and cell line dependent.

\section{Conflict of Interests}

The authors declare that there is no conflict of interests regarding the publication of this paper. 


\section{Acknowledgments}

This work was partially supported by the National Science Foundation (CMMl-1000831). The authors would like to thank the Characterization Center for Materials and Biology (CCMB) at UT Arlington for providing financial and technical support for the electron microscopy characterization.

\section{References}

[1] Z. Fang, C. Lin, R. Ma, S. Huang, and X. Zhu, "Planar plasmonic focusing and optical transport using CdS nanoribbon," ACS Nano, vol. 4, no. 1, pp. 75-82, 2010.

[2] Z. Fang, J. Cai, Z. Yan, P. Nordlander, N. J. Halas, and X. Zhu, "Removing a wedge from a metallic nanodisk reveals a fano resonance," Nano Letters, vol. 11, no. 10, pp. 4475-4479, 2011.

[3] Z. Fang, Q. Peng, W. Song et al., "Plasmonic focusing in symmetry broken nanocorrals," Nano Letters, vol. 11, no. 2, pp. 893897, 2011.

[4] Z. Fang, Y. -R. Zhen, L. Fan, X. Zhu, and P. Nordlander, "Tunable wide-angle plasmonic perfect absorber at visible frequencies," Physical Review B, vol. 85, Article ID 245401, 2012.

[5] G. Han, P. Ghosh, and V. M. Rotello, "Multifunctional gold nanoparticles for drug delivery," in Advances in Experimental Medicine and Biology, vol. 620, pp. 48-56, 2007.

[6] K. K. Jain, "Applications of nanobiotechnology in clinical diagnostic," Clinical Chemistry, vol. 53, no. 11, pp. 2002-2009, 2007.

[7] S. Gelperina, K. Kisich, M. D. Iseman, and L. Heifets, “The potential advantages of nanoparticle drug delivery systems in chemotherapy of tuberculosis," American Journal of Respiratory and Critical Care Medicine, vol. 172, no. 12, pp. 1487-1490, 2005.

[8] O. C. Farokhzad, J. Cheng, B. A. Teply et al., "Targeted nanoparticle-aptamer bioconjugates for cancer chemotherapy in vivo," Proceedings of the National Academy of Sciences of the United States of America, vol. 103, no. 16, pp. 6315-6320, 2006.

[9] S. Dhar, N. Kolishetti, S. J. Lippard, and O. C. Farokhzad, "Targeted delivery of a cisplatin prodrug for safer and more effective prostate cancer therapy in vivo," Proceedings of the National Academy of Sciences of the United States of America, vol. 108, no. 5, pp. 1850-1855, 2011.

[10] G. F. Paciotti, L. Myer, D. Weinreich et al., "Colloidal gold: a novel nanoparticle vector for tumor directed drug delivery," Drug Delivery, vol. 11, no. 3, pp. 169-183, 2004.

[11] G. F. Paciotti, D. G. I. Kingston, and L. Tamarkin, "Colloidal gold nanoparticles: a novel nanoparticle platform for developing multifunctional tumor-targeted drug delivery vectors," Drug Development Research, vol. 67, no. 1, pp. 47-54, 2006.

[12] M. Modo, J. W. M. Bulte, M. Mathias, and J. J. Modo, Nanoparticles in Biomedical Imaging Emerging Technologies and Applications, Springer Science+Business Media, New York, NY, USA, 2008.

[13] A. Dingler, R. P. Blum, H. Niehus, R. H. Müller, and S. Gohla, "Solid lipid nanoparticles (SLN(TM)/Lipopearls(TM))-a pharmaceutical and cosmetic carrier for the application of vitamin E in dermal products," Journal of Microencapsulation, vol. 16, no. 6, pp. 751-767, 1999.

[14] V. L. Colvin, "The potential environmental impact of engineered nanomaterials," Nature Biotechnology, vol. 21, pp. 11661170, 2003.
[15] A. Nel, T. Xia, L. Mädler, and N. Li, "Toxic potential of materials at the nanolevel," Science, vol. 311, no. 5761, pp. 622-627, 2006.

[16] R. D. Averitt, D. Sarkar, and N. J. Halas, "Plasmon resonance shifts of Au-coated $\mathrm{Au}_{2} \mathrm{~S}$ nanoshells: insight into multicomponent nanoparticle growth," Physical Review Letters, vol. 78, no. 22, pp. 4217-4220, 1997.

[17] L. R. Hirsch, R. J. Stafford, J. A. Bankson et al., "Nanoshellmediated near-infrared thermal therapy of tumors under magnetic resonance guidance," Proceedings of the National Academy of Sciences of the United States of America, vol. 100, no. 23, pp. 13549-13554, 2003.

[18] A. M. Gobin, M. H. Lee, N. J. Halas, W. D. James, R. A. Drezek, and J. L. West, "Near-infrared resonant nanoshells for combined optical imaging and photothermal cancer therapy," Nano Letters, vol. 7, no. 7, pp. 1929-1934, 2007.

[19] K. Fu, J. Sun, L. R. Bickford et al., "Measurement of immunotargeted plasmonic nanoparticles' cellular binding: a key factor in optimizing diagnostic efficacy," Nanotechnology, vol. 19, no. 4, Article ID 045103, 2008.

[20] S. Lal, S. E. Clare, and N. J. Halas, "Nanoshell-enabled photothermal cancer therapy: impending clinical impact," Accounts of Chemical Research, vol. 41, no. 12, pp. 1842-1851, 2008.

[21] S. Lal, N. K. Grady, J. Kundu, C. S. Levin, J. B. Lassiter, and N. J. Halas, "Tailoring plasmonic substrates for surface enhanced spectroscopies," Chemical Society Reviews, vol. 37, no. 5, pp. 898-911, 2008.

[22] R. Bardhan, W. Chen, C. Perez-Torres et al., "Nanoshells with targeted simultaneous enhancement of magnetic and optical imaging and photothermal therapeutic response," Advanced Functional Materials, vol. 19, no. 24, pp. 3901-3909, 2009.

[23] J. R. Cole, N. A. Mirin, M. W. Knight, G. P. Goodrich, and N. J. Halas, "Photothermal efficiencies of nanoshells and nanorods for clinical therapeutic applications," The Journal of Physical Chemistry C, vol. 113, no. 28, pp. 12090-12094, 2009.

[24] Y. Wang, W. Qian, Y. Tan, and S. Ding, "A label-free biosensor based on gold nanoshell monolayers for monitoring biomolecular interactions in diluted whole blood," Biosensors and Bioelectronics, vol. 23, no. 7, pp. 1166-1170, 2008.

[25] A. F. Low, G. J. Tearney, B. E. Bouma, and I. K. Jang, “Technology Insight: optical coherence tomography-current status and future development," Nature Clinical Practice Cardiovascular Medicine, vol. 3, pp. 154-162, 2006.

[26] J. Chen, F. Saeki, B. J. Wiley et al., "Gold nanocages: bioconjugation and their potential use as optical imaging contrast agents," Nano Letters, vol. 5, no. 3, pp. 473-477, 2005.

[27] R. A. Jensen, J. Sherin, and S. R. Emory, "Single nanoparticle based optical pH probe," Applied Spectroscopy, vol. 61, no. 8, pp. 832-838, 2007.

[28] S. Keren, C. Zavaleta, Z. Cheng, A. De La Zerda, O. Gheysens, and S. S. Gambhir, "Noninvasive molecular imaging of small living subjects using Raman spectroscopy," Proceedings of the National Academy of Sciences of the United States of America, vol. 105, no. 15, pp. 5844-5849, 2008.

[29] M. Rycenga, Z. Wang, E. Gordon et al., "Probing the photothermal effect of gold-based nanocages with surface-enhanced Raman scattering (SERS)," Angewandte Chemie, vol. 48, no. 52, pp. 9769-9927, 2009. 
[30] M. S. Yavuz, Y. Cheng, J. Chen et al., "Gold nanocages covered by smart polymers for controlled release with near-infrared light," Nature Materials, vol. 8, no. 12, pp. 935-939, 2009.

[31] L. Au, J. Chen, L. V. Wang, and Y. Xia, "Gold nanocages for cancer imaging and therapy," Methods Molecular Biology, vol. 624, pp. 83-99, 2010.

[32] V. P. Zharov, K. E. Mercer, E. N. Galitovskaya, and M. S. Smeltzer, "Photothermal nanotherapeutics and nanodiagnostics for selective killing of bacteria targeted with gold nanoparticles," Biophysical Journal, vol. 90, no. 2, pp. 619-627, 2006.

[33] C. Loo, A. Lowery, N. Halas, J. West, and R. Drezek, "Immunotargeted nanoshells for integrated cancer imaging and therapy," Nano Letters, vol. 5, no. 4, pp. 709-711, 2005.

[34] X. Huang, I. H. El-Sayed, W. Qian, and M. A. El-Sayed, "Cancer cell imaging and photothermal therapy in the nearinfrared region by using gold nanorods," Journal of the American Chemical Society, vol. 128, no. 6, pp. 2115-2120, 2006.

[35] X. Huang, P. K. Jain, I. H. El-Sayed, and M. A. El-Sayed, "Determination of the minimum temperature required for selective photothermal destruction of cancer cells with the use of immunotargeted gold nanoparticles," Photochemistry and Photobiology, vol. 82, no. 2, pp. 412-417, 2006.

[36] X. Huang, W. Qian, I. H. El-Sayed, and M. A. El-Sayed, "The potential use of the enhanced nonlinear properties of gold nanospheres in photothermal cancer therapy," Lasers in Surgery and Medicine, vol. 39, no. 9, pp. 747-753, 2007.

[37] J. Chen, D. Wang, J. Xi et al., "Immuno gold nanocages with tailored optical properties for targeted photothermal destruction of cancer cells," Nano Letters, vol. 7, no. 5, pp. 1318-1322, 2007.

[38] L. Tong, Y. Zhao, T. B. Huff, M. N. Hansen, A. Wei, and J.-X. Cheng, "Gold nanorods mediate tumor cell death by compromising membrane integrity," Advanced Materials, vol. 19, no. 20, pp. 3136-3141, 2007.

[39] W. Cai, T. Gao, H. Hong J, and Sun, "Applications of gold nanoparticles in cancer nanotechnology," Nanotechnology, Science and Applications, vol. 1, pp. 17-32, 2008.

[40] B. Merchant, "Gold, the Noble metal and the paradoxes of its toxicology," Biologicals, vol. 26, no. 1, pp. 49-59, 1998.

[41] C. Huang, Y. Hao, J. Nyagilo, D. P. Dave, L. Xu, and X. Sun, "Porous Hollow gold nanoparticles for cancer SERS imaging," Journal of Nano Research, vol. 10, pp. 137-148, 2010.

[42] C. Huang, J. Jiangs, M. Lu, L. Sun, E. I. Meletis, and Y. Hao, "Capturing electrochemically evolved nanobubbles by electroless deposition. A facile route to the synthesis of hollow nanoparticles," Nano Letters, vol. 9, no. 12, pp. 4297-4301, 2009.

[43] Y. Sun and Y. Xia, "Shape-controlled synthesis of gold and silver nanoparticles," Science, vol. 298, no. 5601, pp. 2176-2179, 2002.

[44] N. R. Jana, L. Gearheart, and C. J. Murphy, "Wet chemical synthesis of high aspect ratio cylindrical gold nanorods," The Journal of Physical Chemistry B, vol. 105, no. 19, pp. 4065-4067, 2001.

[45] E. C. Cho, Q. Zhang, and Y. Xia, "The effect of sedimentation and diffusion on cellular uptake of gold nanoparticles," Nature Nanotechnolgy, vol. 6, pp. 385-391, 2011.

[46] B. C. Heng, X. Zhao, S. Xiong, K. W. Ng, F. Y.-C. Boey, and J. S.-C. Loo, "Cytotoxicity of zinc oxide $(\mathrm{ZnO})$ nanoparticles is influenced by cell density and culture format," Archives of Toxicology, vol. 85, no. 6, pp. 695-704, 2011.
[47] C. Uboldi, D. Bonacchi, G. Lorenzi et al., "Gold nanoparticles induce cytotoxicity in the alveolar type-II cell lines A549 and NCIH441," Particle and Fibre Toxicology, vol. 6, article 18, 2009. 

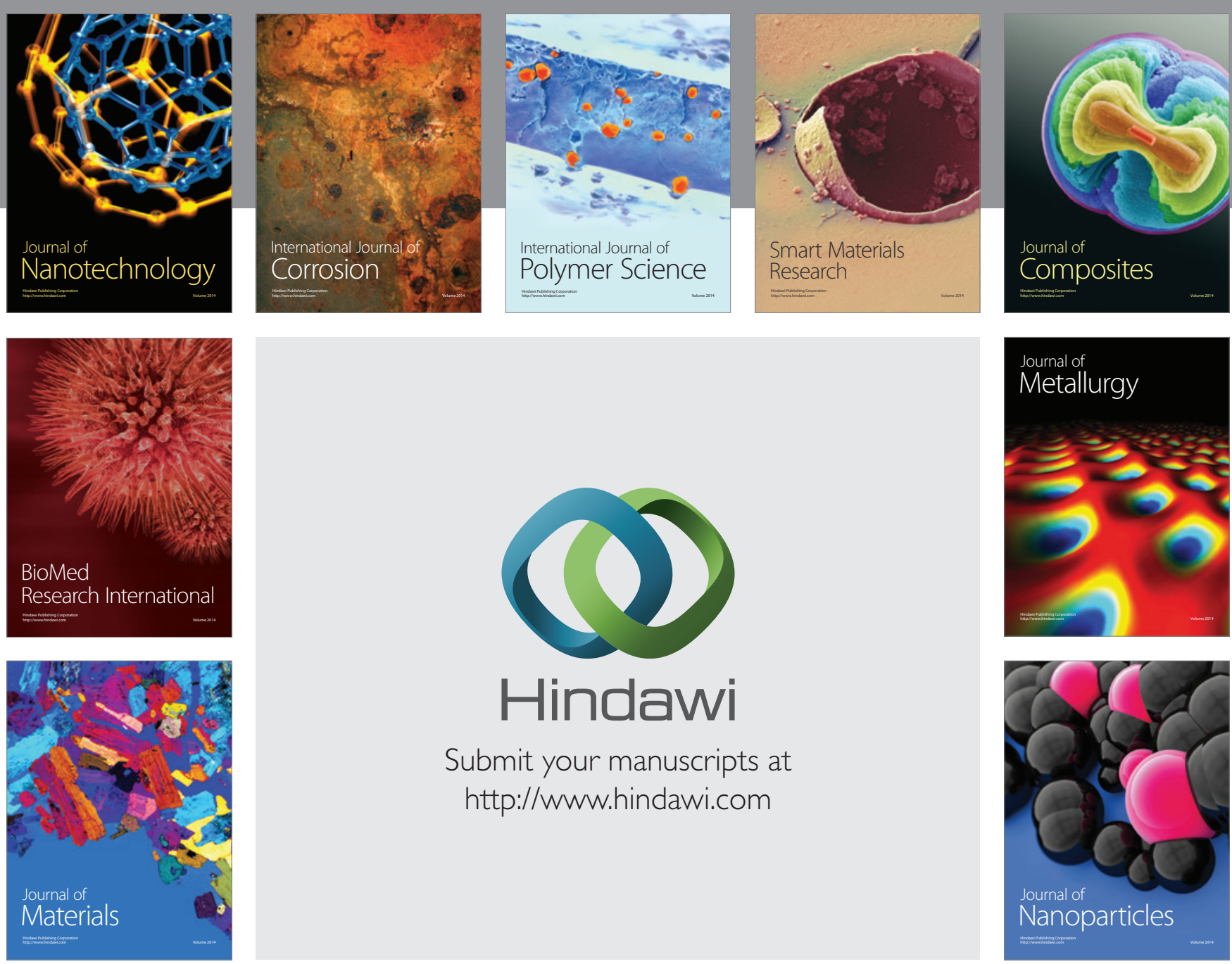

Submit your manuscripts at http://www.hindawi.com
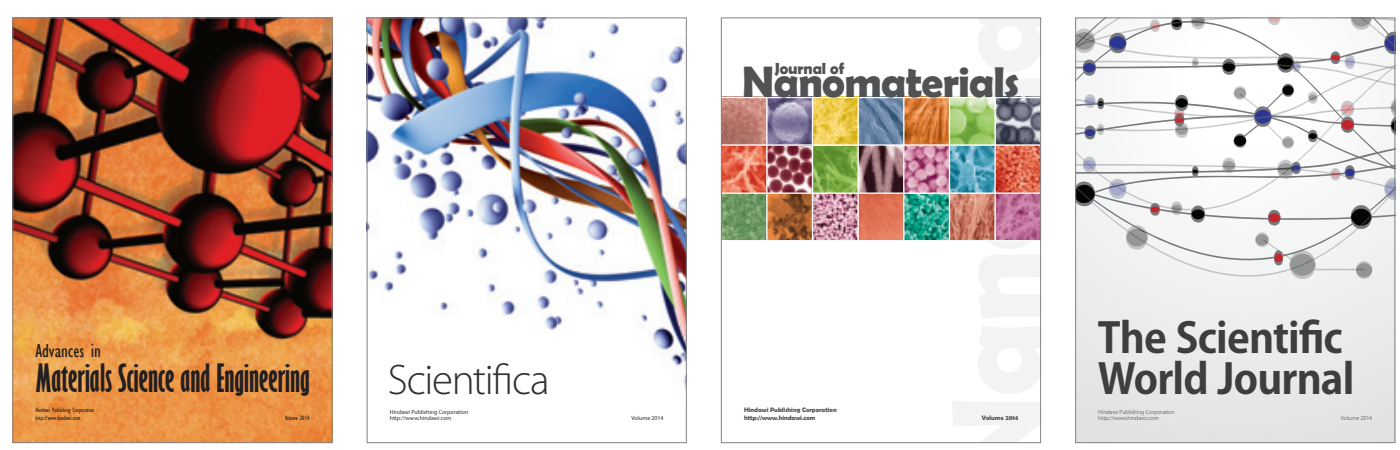

\section{The Scientific World Journal}
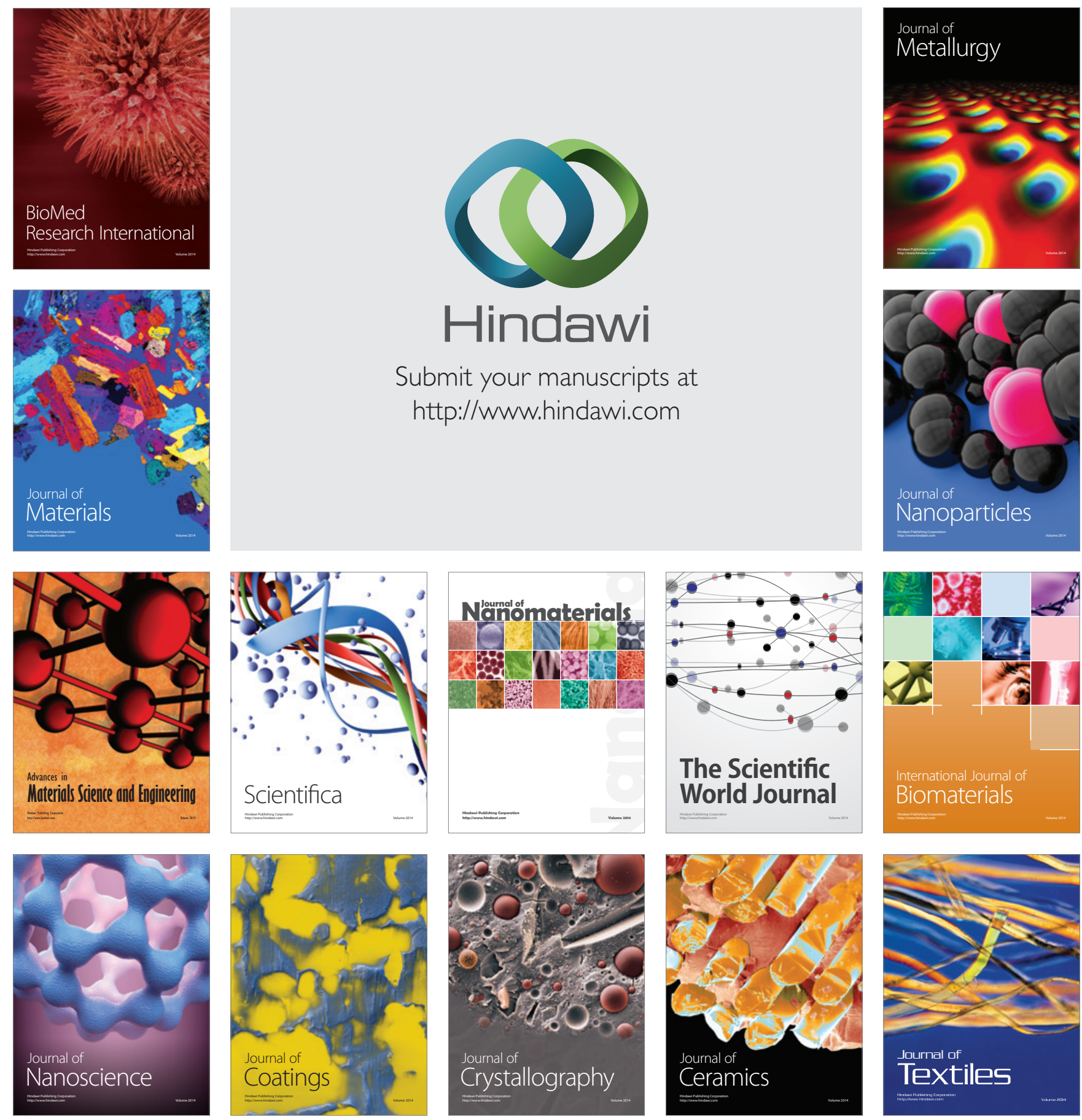